\title{
Recent Developments on Fixed Point Theory in Function Spaces and Applications to Control and Optimization Problems
}

\author{
Hemant Kumar Nashine, ${ }^{1}$ Mujahid Abbas, ${ }^{2}$ Marlène Frigon, ${ }^{3}$ and Calogero Vetro ${ }^{4}$ \\ ${ }^{1}$ Department of Mathematics, Disha Institute of Management and Technology, Satya Vihar, Vidhansabha-Chandrakhuri Marg, \\ Mandir Hasaud, Raipur, Chhattisgarh 492101, India \\ ${ }^{2}$ Department of Mathematics and Applied Mathematics, University of Pretoria, Private Bag X20, Hatfield, Pretoria 0028, South Africa \\ ${ }^{3}$ Département de Mathématiques et de Statistique, Université de Montréal, CP 6128, Succursale Centre-Ville, \\ Montréal, QC, Canada H3C 3J7 \\ ${ }^{4}$ Department of Mathematics and Informatics, University of Palermo, Via Archirafi 34, 90123 Palermo, Italy
}

Correspondence should be addressed to Hemant Kumar Nashine; drhknashine@gmail.com

Received 20 April 2015; Accepted 20 April 2015

Copyright (C) 2015 Hemant Kumar Nashine et al. This is an open access article distributed under the Creative Commons Attribution License, which permits unrestricted use, distribution, and reproduction in any medium, provided the original work is properly cited.

Nonlinear and Convex Analysis have as one of their goals solving equilibrium problems arising in applied sciences. In fact, a lot of these problems can be modelled in an abstract form of an equation (algebraic, functional, differential, integral, etc.), and this can be further transferred into a form of a fixed point problem of a certain operator. In this context, finding solutions of fixed point problems, or at least proving that such solutions exist and can be approximately computed, is a very interesting area of research.

The Banach Contraction Principle is one of the cornerstones in the development of Nonlinear Analysis, in general, and metric fixed point theory, in particular. This principle was extended and improved in many directions and various fixed point theorems were established. Two usual ways for extending and improving the Banach Contraction Principle are obtained by (1) changing the contraction condition to more general ones and (2) replacing the complete metric space by certain generalized metric spaces.

In this special issue we focused on applications in the area of nonlinear functional analysis, in particular to control and optimization problems. We received submissions devoted to the study of fixed points and fixed point spaces with applications; fractional evolution equations with applications; operator equations; best approximation theorems in abstract spaces; convergence and stability of iteration procedures; semilinear control problems.
Now, we are going to describe briefly the papers published in the special issue.

B. Alamri et al. discussed the completeness of $v$ generalized metric spaces in the sense of Branciari. Also, they generalized Subrahmanyam's and Caristi's fixed point theorems.

N. I. Mahmudov and M. A. McKibben studied the approximate controllability of fractional evolution equations involving generalized Riemann-Liouville fractional derivative. To obtain their results, the authors used the theory of fractional calculus, semigroup theory, and the Schauder fixed point theorem under the assumption that the corresponding linear system is approximately controllable.

M. A. Kutbi et al. introduced new concepts of $\alpha_{c}$ $G F$-contractive non-self-mapping, weak $\alpha_{c}$-GF-contractive non-self-mapping, generalized $\alpha_{c}$-GF-contractive non-selfmapping, and Suzuki type GF-contractions. Then, they established the existence of $P P F$ dependent fixed point theorems for such kind of contractive non-self-mappings in the Razumikhin class. They used these results to deduce some PPF dependent fixed point theorems for GF-contractive non-selfmappings, whenever the range space is endowed with a graph or a partial order.

M. De la Sen and E. Karapinar discussed the properties of convergence of distances of $p$-cyclic contractions on the union of the $p$ subsets of an abstract set $X$ defining 
probabilistic metric spaces and Menger probabilistic metric spaces as well as the characterization of Cauchy sequences which converge to best proximity points. The existence and uniqueness of fixed points and best proximity points of $p$ cyclic contractions, defined in induced complete Menger probabilistic metric spaces, are also discussed in the case that the associate complete metric space is a uniformly convex Banach space. Finally, the fixed points of the $p$-composite mappings restricted to each of the $p$ subsets in the cyclic framework disposal are investigated.

$\mathrm{K}$. Wongkum et al. worked on the generalized UlamHyers-Rassias stability of a quadratic functional equation, by using methods of fixed point theory in the framework of modular spaces whose modulars are lower semicontinuous but do not satisfy any relatives of $\Delta_{2}$-conditions.

We hope that the results contained in this special issue will create the inspiration for researchers working in fixed point theory and its applications to differential, integral, and functional equations.

\section{Acknowledgment}

All the editors of this special issue would like to express their gratitude to the authors of all papers submitted for consideration.

Hemant Kumar Nashine

Mujahid Abbas Marlène Frigon

Calogero Vetro 


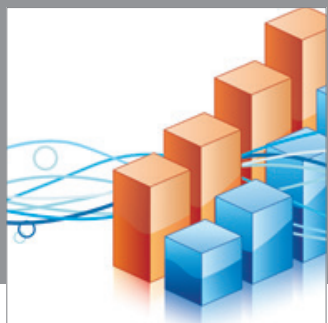

Advances in

Operations Research

mansans

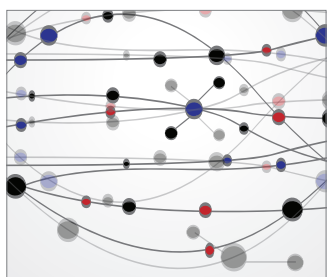

The Scientific World Journal
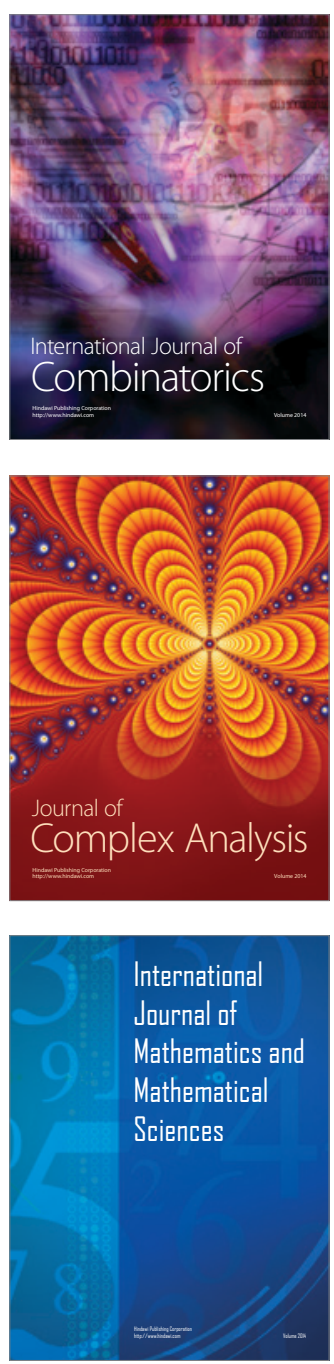
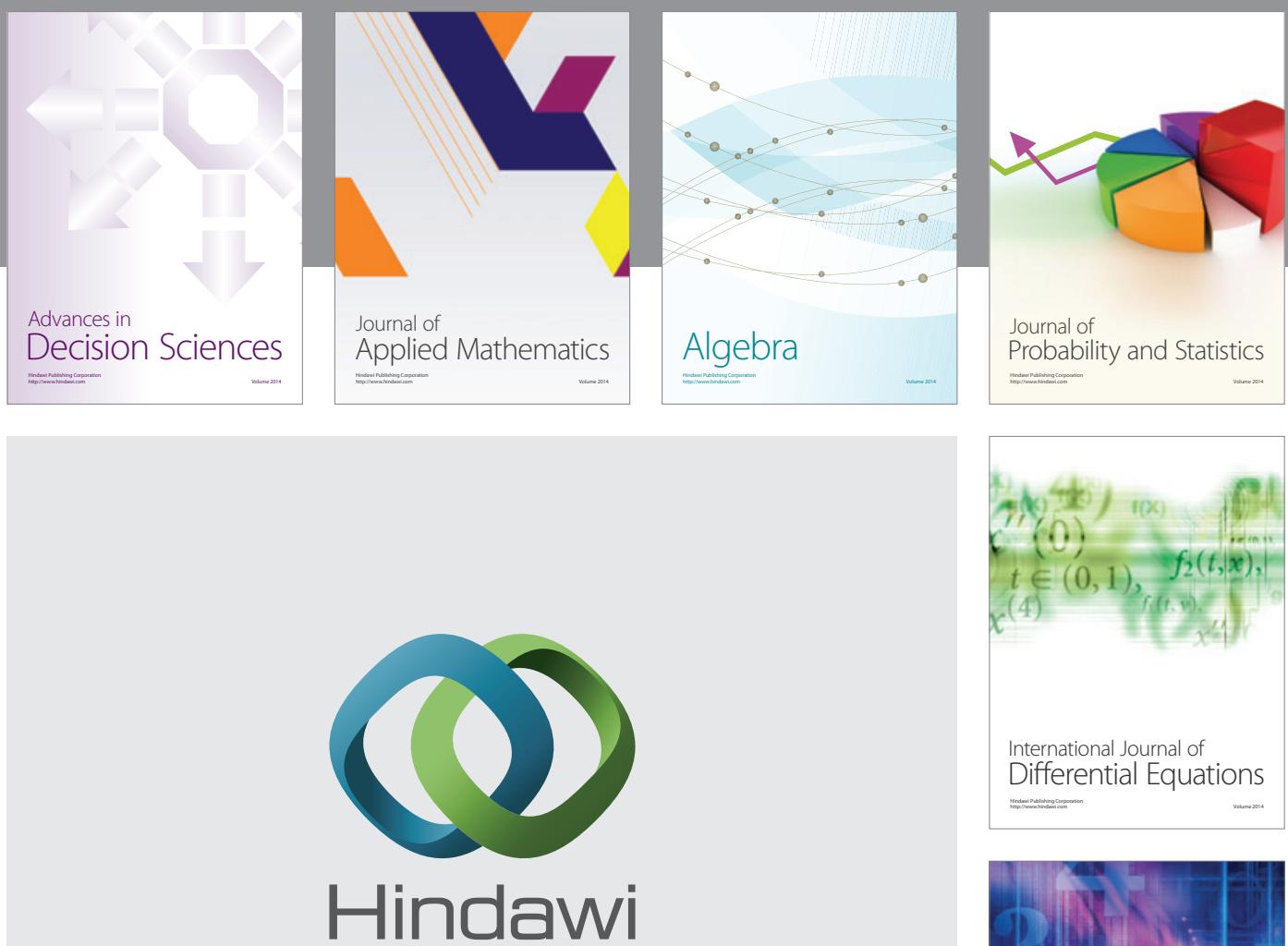

Submit your manuscripts at http://www.hindawi.com
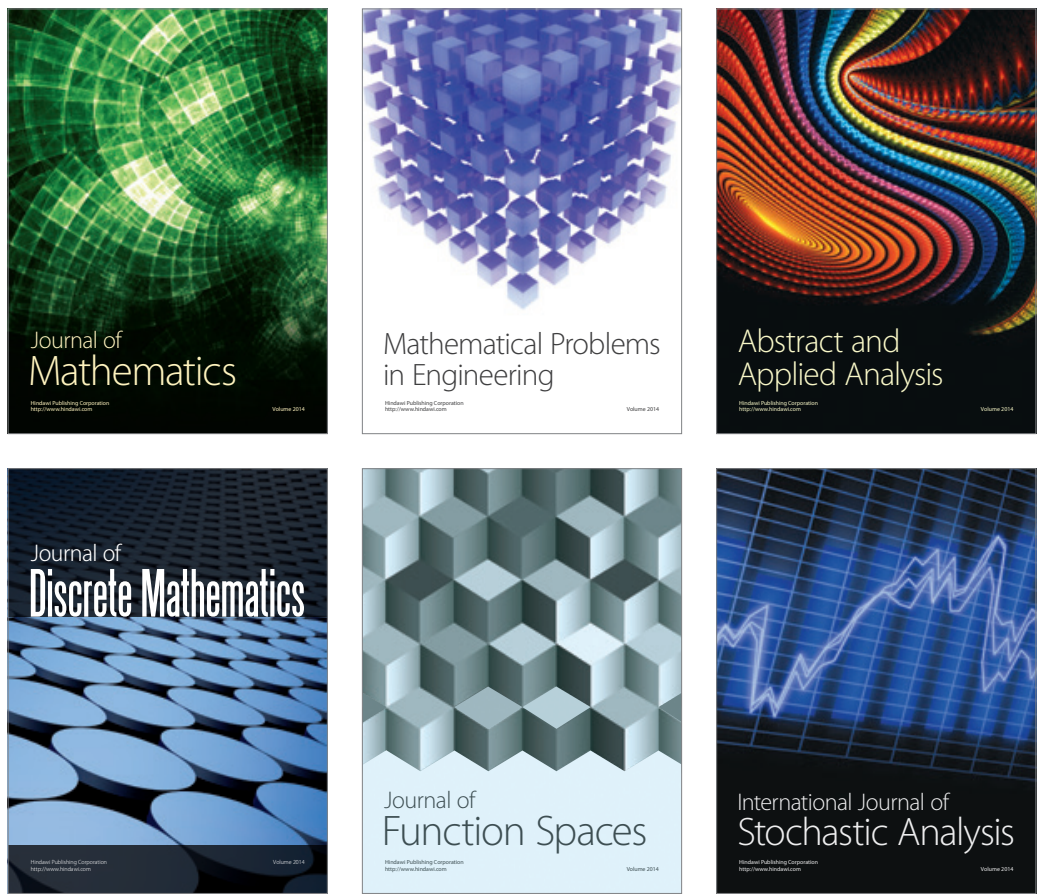

Journal of

Function Spaces

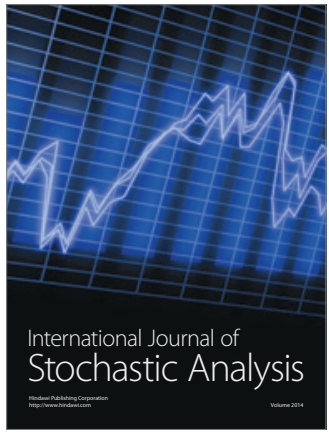

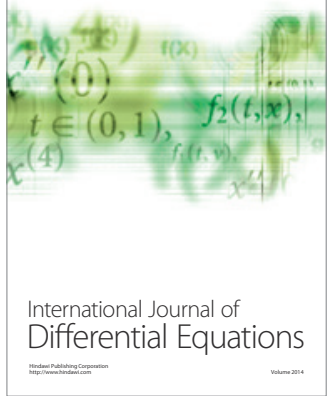
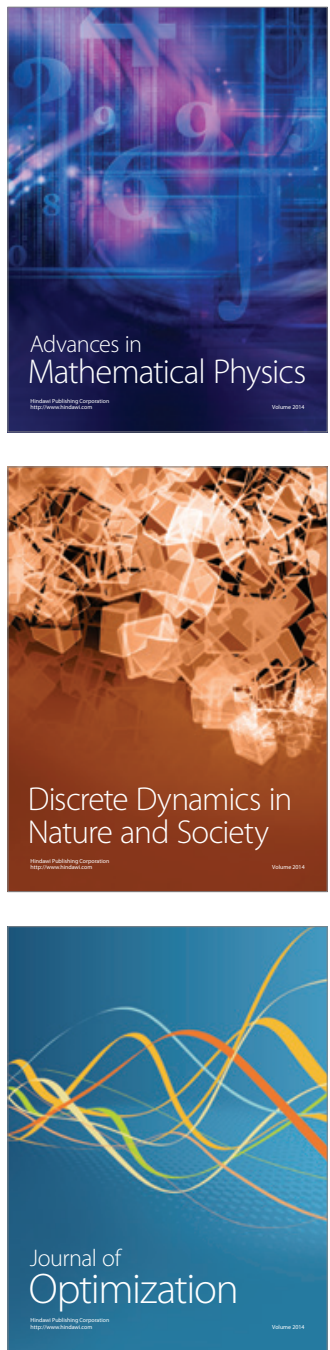